\title{
Ingenio e innovación. Los atrapanieblas como objetos-fluidos
}

Joaquín YRIVARREN

\begin{abstract}
RESUMEN
En este ensayo ponemos en discusión la noción de 'prospectiva' y los dilemas de imaginar el futuro para tomar decisiones hoy. Para muchos la vida cotidiana es experimentada dentro del estilo del corto plazo ('acontecimientos'), mientras la prospectiva apunta a un horizonte abierto y múltiple para acciones y proyectos ('escenarios'). El problema es precisamente encontrar un buen ensamblaje entre acontecimientos y escenarios, o también, entre el ingenio y la innovación. Nuestra propuesta, ampliamente, es reconocer los escenarios (mundos posibles y deseados) en los acontecimientos y asentar la innovación en los recursos y aprendizajes introducidos por el ingenio. Empíricamente, ello conlleva analizar 'datos portadores de (poco) futuro' y (pluri)tendecias. Este ensayo se desenvuelve en torno al caso de los atrapanieblas en el distrito de Villa María del Triunfo (Lima), describiendo el tránsito desde su experimentación cerrada, su definición como 'objeto-fluido' y su incorporación en un proyecto urbano más amplio.
\end{abstract}

Palabras ClaVe: Prospectiva, ingenio, innovación, atrapanieblas, objeto-fluido.

\section{Ingenuity and innovation. The catch-fogs as objects-fluids}

\begin{abstract}
In this essay we discuss the notion of 'prospective' and the dilemmas to imagine the future for taking decisions now. For many, daily life follows a short-term horizon ('events'), meanwhile the 'prospective' looks for a new open horizon for actions and projects ('scenarios'). The matter is, precisely, to articulate 'events' and 'scenarios', or which is the same, to traslate ingenious projects into long-term innovations. Our broader aim is to describe possible worlds without forgetting 'events', as well as to base innovation into resources and learnings steammed from ingenuity. This aim, empirically, implies to analyze 'data of little future' and 'pluri-trends'. For, we present the case of catch-fogs in Villa María del Triunfo (Lima) and describe the transit from its period of trials, its definition as 'fluid-object' until its growth as an urban project.
\end{abstract}

KEYWORDS: Prospective, ingenuity, innovation, catch-fogs.

ISSN 1605-8933 | 25(2015)129-145 I unMSM, LIMA, PERúl REVISTA DE SOCIOLOGIIA 25 /129 
En lugar del lema un tanto engañoso 'la verdad nos hará libres', deberíamos decir nuestra capacidad para volver a describir las cosas con términos novedosos nos hará más ricos, complejos e interesantes de lo que éramos.

(RICHARD RORTY, Filosofía y Futuro, p. 11)

\section{Introducción}

- ste ensayo nace en virtud de un breve trabajo de investigación acerca de la noción de 'prospectiva', noción que es utilizada por el Centro Nacional - de Planificación Estratégica (Ceplan)². La prospectiva propone imaginar el futuro para tomar decisiones hoy. Quizá ello pueda sonar inhabitual en un país que problemas ha tenido y tiene para reconciliar el recuerdo del pasado con la acción del presente. La descripción de un tiempo compartido en nuestro país ha sido tarima para la reproducción de desigualdades, antes que recurso para el reconocimiento mutuo. Tenemos una 'historia oficial' que por mucho tiempo olvidó a pueblos indígenas y amazónicos, por ejemplo; o una historia reciente del conflicto armado interno que algunos sectores pretenden olvidar, pasando la página (ej. El amplio rechazo al Museo de la Memoria es elocuente).

También podría parecer extraña la prospectiva cuando identificamos que el origen de la creatividad, sobre todo en lo que toca a oficios y artes ingeniosos, es concurrente a la precariedad de la vida cotidiana. A más de ello, sabemos bien de las dificultades de las instituciones públicas y privadas para reconocer, en el largo plazo, aquellas sensibilidades fermentadas día a día. En este sentido, una propuesta que apunta a un tiempo compartido y proyectado a futuro, así como a una conexión entre imaginación e instituciones merece una reflexión pública como la que deseamos hacer aquí.

La prospectiva entendámosla, sencillamente, como una herramienta para pensar y explorar mundos posibles y, en base a ellos, tomar decisiones y actuar en el presente (Bas 2004, Bitar 2014). El primer dilema que identificamos en la prospectiva es, precisamente, la posibilidad de articulación de los acontecimientos presentes con la fabricación de escenarios futuros. Dicho en otras palabras, lograr sintonizar lo que la gente hace y los deseos con que infunde sus proyectos (lo que denominaremos 'acontecimientos'), por un lado, y lo que, por otro, los expertos trazan como tendencias a largo plazo (las llamaremos también, 'escenarios').

1 En agosto de 2014 me fue encargado un trabajo de investigación por el Ceplan: “América Latina: imaginando el futuro hoy." Para este ensayo tomamos solo la última parte del documento final, revisada y ajustada para la presente publicación.

\section{0/ REVISTA DE SOCIOLOGÍA 25}


Comprender y resolver este dilema es importante para asentar un sentimiento de confianza en los proyectos que emprendemos como individuos y organizaciones. De hecho, la finalidad de la planificación nacional es la generación de confianza, entendida como una capacidad para elaborar hipótesis razonables sobre el comportamiento de los demás y sobre la continuidad de los proyectos. Siendo un conjunto de 'hipótesis razonables', la confianza no puede sostenerse ni en la certeza absoluta, ni en el más crudo desconocimiento; se ubica, más bien, en una suerte de zona intermedia, donde proyectarse hacia afuera requiere un clima de coherencia y respaldo incluso en el error. Como sostuviera G. Simmel (1972): quien conoce mucho no puede confiar, lo mismo que quien ignora demasiado. Por esto, es conveniente poner entre paréntesis la idea heredada de que los expertos conocen y mucho y que la gente ingnora demasiado; idea que justifica ceder la tarea de elaborar imágenes fiables del futuro a los expertos. El problema de mantener en pie los extremos de la ciencia y la sociedad - como en el mito de la caverna de Platón - es que ello inhibe el sentimiento de confianza mutua.

A veces, el apuro por influir sobre las decisiones públicas lleva a los expertos en prospectiva a las orillas de la certeza, alejándolos de la experimentación. $Y$ en consonancia, en su discurso se llega a sentir una ambición competitiva antes que un deseo por componer un tiempo compartido. Ello se nota en un recurso retórico frecuentemente usado al hablar de las (mega)tendencias a futuro: "así van a ser las cosas, ¿estamos preparados?" Esta frase recurrente asume como cierto un estado de cosas en el mundo, a nivel de los hechos, $y$ agrega juntamente una cuota de ansiedad, a nivel de las emociones. Certeza y ansiedad no son, a nuestro juicio, una buena combinación.

Asimismo, en el discurso prospectivo un elemento clave son los llamados "datos portadores de futuro", datos en base a los cuales se van fabricando las (mega)tendencias, como los expertos suelen denominar también a los escenarios a futuro. La característica de estos datos es que conforman enunciados poco-polémicos, es decir, enunciados que no hacen explícito quiénes, cuándo, cómo ni dónde fueron elaborados esos datos. En nuestro argumento, en cambio, el pensamiento a largo plazo se benificiaría de la discusión sobre “datos portadores de (poco) futuro", por cuanto con ellos seríamos más concientes de las redes de producción de conocimientos y artefactos, y porque ellos nos atarían a los acontecimientos y proyectos cotidianos. Una mayor conciencia de estos acontecimientos podría habilitar una mejor representación del futuro deseado, en el sentido de columbrar (pluri)tendencias en lugar de (mega)tendencias. Las (pluri)tendencias, como veremos, se parecen más a 
preguntas experimentales que se van formulando y cambiando en medio de la trayectoria de un proyecto.

En este ensayo buscamos rastrear y describir una zona intermedia entre acontecimientos y escenarios, una zona compuesta de datos portadores de (poco) futuro y (pluri)tendencias. Nuestro supuesto, ciertamente discutible, es que desde allí se puede ampliar un sentimiento de confianza y coherencia básicas porque es una zona de enunciados experimentales (no-ceterza) que van madurando lentamente (no ansiedad). Sometemos a examen, de modo prelimirar, un experimento social llamado "atrapanieblas", que sirve como ejemplo de un proyecto que poco a poco fue sumando acontecimientos ingeniosos y ampliando escenarios de innovación.

\section{Entre acontecimientos y escenarios: datos portadores de (poco) futuro}

El reto de la prospectiva es incorporar en el debate público un nuevo horizonte de la acción: el futuro como escenario múltiple y abierto. Pero, ¿desde dónde y cómo inicar a fabricarlo? Uno de los dilemas a este respecto es llevar a la práctica un vínculo alternativo entre ciencia y sociedad, entre los escenarios representados por expertos y los acontecimientos de la vida cotidiana. Las más de las veces no sintonizan unos y otros; incluso, pareciera que la escala con la que el tiempo se presenta en ambos casos es diversa o contradictoria. Por ello, consideramos útil buscar la forma de reconectar el tiempo compartido a largo plazo y la sensibilidad pública fermentada en proyectos portadores de (poco) futuro.

Los escenarios suelen ser representaciones y cuantificaciones de patrones de acción que pierden su carácter local y contextual, para habilitar una visión más global y estándar de tendencias, riesgos y posibilidades. Los escenarios, tal lo propone metodología prospectiva (método Délfos), suelen ser además el producto de consenso de una asamblea de expertos (Bas 2004). La cotidianidad en casi todo el espectro social peruano, en contraste, suele ser vivida dentro de los límites del corto plazo. Para mucha gente en nuestro país el futuro se siente como un horizonte cerrado. Las tendencias, posibilidades y riesgos son más difíciles de dilucidar.

En un manuscrito inédito titulado "Vindicación del ingenio. Acerca de la vida dura y el crecimiento moral” (2005) ${ }^{2}$, el sociólogo G. Nugent carateriza el corto

2 Manuscrito citado con consentimiento del autor. Los fragmentos incluidos en este documento de trabajo pertenecen a la sección titulada “El corto plazo”, incluida en la primera parte del libro llamada "Escenarios".

\section{2/ REVISTA DE SOCIOLOGÍA 25}


plazo como un estilo de vida no limitado a personas en condición de pobreza, sino transversal al escenario social peruano. Al ser tratado como un estilo, el corto plazo no sería una normativa que termina constriñendo la vida social, ni tampoco pertenecería al terreno de la acción individual conciente. Antes bien, un estilo es un "supuesto práctico para hacer cosas"; pertence a lo que solemos llamar conocimientos tácitos o hábitos. Y es precisamente esta dimensión habitual de la vida la que exige conectarse a un horizonte múltiple y abierto.

Para comprender mejor el estilo del corto plazo en nuestro país conviene diferenciarlo de la sensación derivada de la 'incertidumbre', es decir, aquella sensación derivada de las consecuencias imprevistas de las decisiones presentes o asociada a la relatividad de proposiciones consideradas verdaderas (como en el caso de los riesgos ambientales). Por oposición, el corto plazo consiste en un estado de 'transitoriedad' más que de incertidumbre. La transitoriedad es un elemento inhibitorio de la coherencia y el respaldo, tanto en lo que toca a la conformación de la biografía individual, cuanto en lo que atañe a las narrativas colectivas (esas imágenes y descripciones que hacemos de nosotros como nación, ciudad, partido, cultura, etc.).

\begin{abstract}
... un trasfondo de angustia, de inminencia de un colapso en el más extremo de los casos y, en el mejor, una sensación opresiva de transitoriedad. No es el caso de una modernidad vertiginosa donde todo cambia en nombre de un futuro abierto. Aquí la transitoriedad es una consecuencia de un futuro cerrado. Nada de lo que se haga puede tener un sentido razonable de permanencia. Esta presencia del corto plazo no solamente funciona para las situaciones de marcada pobreza, abarca todos los grados del espectro social. (Nugent 2005: s/p)
\end{abstract}

En efecto, el problema es la persistencia de un futuro cerrado en la vida cotidiana, el cual puede ser observado sin gran esfuerzo en descripciones transitorias de la persona, en la multitud de objetos 'parchados' y en las soluciones 'por mientras' que caracterizan parte de nuestra cultura pública. Si bien los estados de permanencia anquilosada, ya se trate a nivel de pensamientos, acciones u objetos, suelen ser estados reacios a la innovación y al cambio; la permanencia, no obstante ello, también podría ser entendida razonablemente como un tiempo institucional que va más allá del día a día, como un mecanismo de coherencia y respaldo incluso en el error y el fracaso. Cuando los individuos y colectividades no poseen este estado de razonable permanencia, el futuro se clausura, y lo que queda es la sensación de precariedad en la que se desenvuelve gran parte del trabajo cotidiano formal e informal. 
Muchos de los que consideramos oficios y artefactos ingeniosos comparten esa existencia parchada o 'por mientras'. Quiero llamar la atención del lector sobre este desfase entre la persistencia del corto plazo que caracteriza nuestro ingenio, y el respaldo a largo plazo que requiere todo desempeño innovador. Resolver ese desequilibrio es uno de los desafíos más arduos de la desigualdad en nuestro país. Un tránsito suave entre los acontecimientos ingeniosos y los escenarios de innovación es sin sombra de duda la base del 'crecimiento moral' (Nugent 2005, Rorty 2002).

Ahora bien, la Imagen 1 es todavía parte de la escenografía de nuestra ciudad, y describe la persistencia del estilo del corto plazo. Y es, además, un ejemplo adecuado de lo que llamaremos un 'dato portador de (poco) futuro', desde donde pretendemos levantar la descripción (o lo que es lo mismo, fabricación) de mundos posibles. Aquellos fierros salientes de un segundo piso son un dato que registra una expectativa, el trazo inicial de un deseo, una proyección a largo plazo pero todavía como 'algún día será' (Nugent 2005). Hay en ellos, podemos sugerir, una demanda latente de prospectiva. Pero, a diferencia de la retórica que estipula los datos de futuro como un "así serán las cosas, ¿estamos preparados?”, consideramos que los datos portadores de (poco) futuro hacen explícita una zona intermedia. En esta zona intermedia no hay seguridades absolutas sobre cómo serán las cosas ni tampoco se disponen de todos los recursos e instrumentos para estar preparado.

En suma, tratamos de poner en evidencia una paradoja entre lo abierto y lo cerrado, entre dos temporalidades contrastantes. Una que traza un horizonte múltiple de largo plazo y otra que reproduce la transitoriedad del corto plazo. El (des)encuentro del largo y el corto plazo puede ser apreciado como un desafío, el cual obliga a ensamblar innovación e ingenio en el Perú. Dicho reensamblaje es posible si visibilizamos, como es nuestra propuesta, zonas intermedias entre los escenarios y los acontecimientos, esto es, si concentramos nuestra atención en investigar 'datos portadores de (poco) futuro' que señalen imperfectamente mundos deseados y posibles y que dejen traslucir su redes de producción de conocimientos y artefactos.

\section{Del ingenio a la innovación: atrapanieblas.}

Sobre este punto, nos gustaría esbozar el caso de los atrapanieblas en nuestra ciudad. En la edición de marzo de 2014, la revista "Arkinka - Revista de Arquitectura, Diseño y Construcción” muestra los proyectos ganadores del Concurso Juan Günther, auspiciado por la Municipalidad de Lima con el ob-

\section{4/ REVISTA DE SOCIOLOGÍA 25}




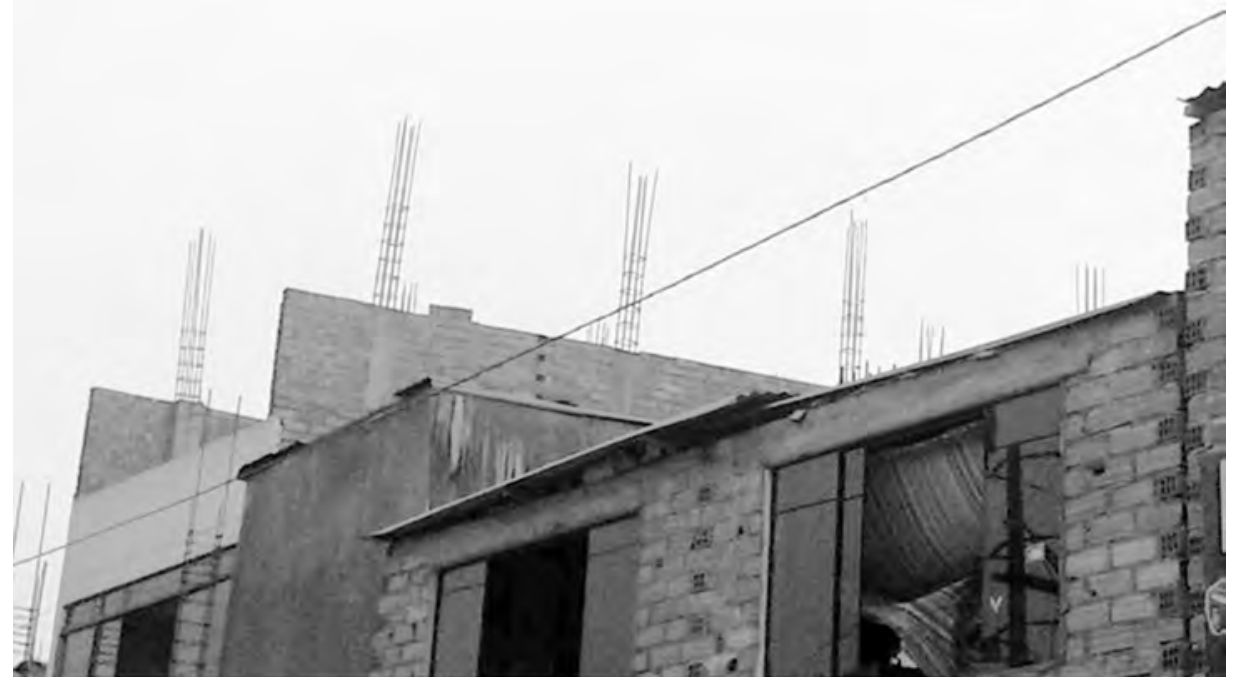

Figura 1: El estilo del corto plazo. Fuente: elaboración propia, tomada en julio de 2014, Surquillo. Nótese que al costado de la casa con los fierros salientes encontramos la figura familiar de una fachada sin tarrajear.

jetivo de imaginar la ciudad a futuro. Digámoslo así, era como un premio a la prospectiva en el campo artquitectónico. El ganador en la categoría Proyectos arquitectónicos para el desarrollo de la cultura en Lima, fue un proyecto llamado "Parque Atrapanieblas", del arquitecto Marius Ege (ver Imagen 4). El diseño del proyecto es altamente pertinente para nuestra reflexión sobre el ensamblaje entre acontecimientos y escenarios. El proyecto traduce un artefacto ingenioso (atrapanieblas en las lomas de Villa María del Triunfo adonde no arriba la red pública de agua) en una innovación urbana. Nuestra idea es que para lograr un tránsito suave del ingenio a la innovación no tenemos porqué operar una ruptura entre acontecimientos y escenarios.

Aquella buena idea no nacio en 2014, en el marco de un concurso de prospectiva; más bien, se remonta varios años atrás, cuando algunos ingenieros y profesionales indagaban sobre algo llamado 'tecnología intermedia' para el manejo del agua de niebla.

En los últimos años, grupos de investigadores en tecnología intermedia, han desarrollado estrategias de bajo costo, experimentando con el agua de niebla por medio de módulos provistos de mallas de nylon, poliester y propileno a los cuales se ha denominado 'atrapanieblas'. (Márquez 1993: 91) 
Subrayemos la definición de los atrapanieblas como tecnologías intermedias. En este sentido, si bien no constituirían obras de gran envergadura con mucha ciencia detrás, tampoco dejarían de ser objetos de investigación y experimentación colectiva. En estos términos, se adecúan a lo que solemos llamar artefactos ingeniosos.

El funcionamiento de estos artefactos ingeniosos consiste en el aprovechamiento del agua de niebla en la ciudad. No se trata de un proceso de condensación, como podría pensarse, sino propiamente de la captura de la niebla, a través de mallas de material diverso, las cuales transforman la sobresaturación de agua en el aire por debajo del punto de rocío en gotas recogidas a través de canaletas ubicadas en la parte inferior de las mallas. Inicialmente, estos artefactos fueron imaginados para la reforestación de las lomas costeñas, para lo cual deberían funcionar entre los 300 y los $1800 \mathrm{msnm}$ y captar entre 1 a 30 litros por metro cuadrado de malla al día $\left(\mathrm{l} / \mathrm{m}^{2} / \mathrm{d}\right)$. He aquí la proyección de un mundo deseado: lomas costeñas más verdes. Hace veinte años, no obstante, las mediciones sobre la ubicación, captación y resistencia aún eran incipientes (Márquez 1993). Es decir, la imaginación de un mundo deseado contrastaba con el bajo nivel de desarrollo de la experimentación.

En una investigación experimental en las lomas de Atocongo entre 1992 y 1993, Pinche y Ruiz (1995) se propusieron determinar cuantitativamente la captación de agua de niebla obtenida con los atrapanieblas, así como examinar la aptitud del agua capturada. Hacemos notar que los ingenieros, a la sazón, dieron por sentado un modelo tipo de atrapaniebla, como se puede leer en esta cita: "Al respecto, los experimentos efectuados desde los años 40 determinaron que el modelo bidimensional y la malla raschell son los más adecuados para este fin." (Pinche y Ruiz 1995: 86). Ahora bien, el experimento que nos interesa describir consistió en las siguientes actividades:

- La instalación de dos estaciones de captación con sendos atrapanieblas de $19 \mathrm{~m}^{2}$ entre noviembre de 1992 y mayo de 1993, a los 400 y $535 \mathrm{msnm}$.

- El análisis de suelo, cuyas muestras fueron analizadas en el laboratorio de suelos del Instituto Nacional de Investigación Agroindustrial del Ministerio de Agricultura.

- El análisis de calidad del agua, a nivel fisico-químico y microbiológico, realizado en el laboratorio de contaminación ambiental y de recursos marinos de la Universidad Federico Villarreal.

- El análisis de aplicabilidad de agua de niebla: para fines forestales y agrícolas, así como para consumo humano.

\section{6/ REVISTA DE SOC1OLOGíA 25}


Los resultados indicaron que durante ese período, que coincide con la finalización del invierno, se obtuvo una captación máxima de $15.55 \mathrm{l} / \mathrm{m}^{2} / \mathrm{d}$ (en noviembre) y una mínima de $0.23 \mathrm{l} / \mathrm{m}^{2} / \mathrm{d}$ (en marzo). El agua obtenida no mostró microorganismos dañinos, pero sí “...el color fue de un matiz marrón claro, con elevada turbiedad...” (1995: 91) y, a más de ello, hubo rastros de exposición a fuentes cercanas de contaminación. De suerte que, para el consumo humano el agua requeriría cierto tratamiento. Asimismo, los autores de la investigación recomendaban un estudio más amplio sobre la rentabilidad y aceptabilidad de la tecnología por parte de la población.

El punto que queremos hacer notar aquí es que, como los fierros de contrucción saliendo de un segundo piso, los atrapanieblas imaginan un mundo deseado: la reforestación de las lomas costeñas. Pero, esta imaginación se presenta aún más como un paquete de preguntas que como respuestas definitivas: ¿El período de noviembre a mayo es el más apropiado para la captura de agua?, ¿el color es solo un problema estético o tendrá que ver con la aceptabilidad?, ¿cómo depurar los nitritos que dejan huella de contaminación en el agua?, ¿serán rentables esos artefactos? Justamente, este es el estado que caracteriza las (pluri)tendencias y que traza el nodo inicial de una cartografía de la tecnología.

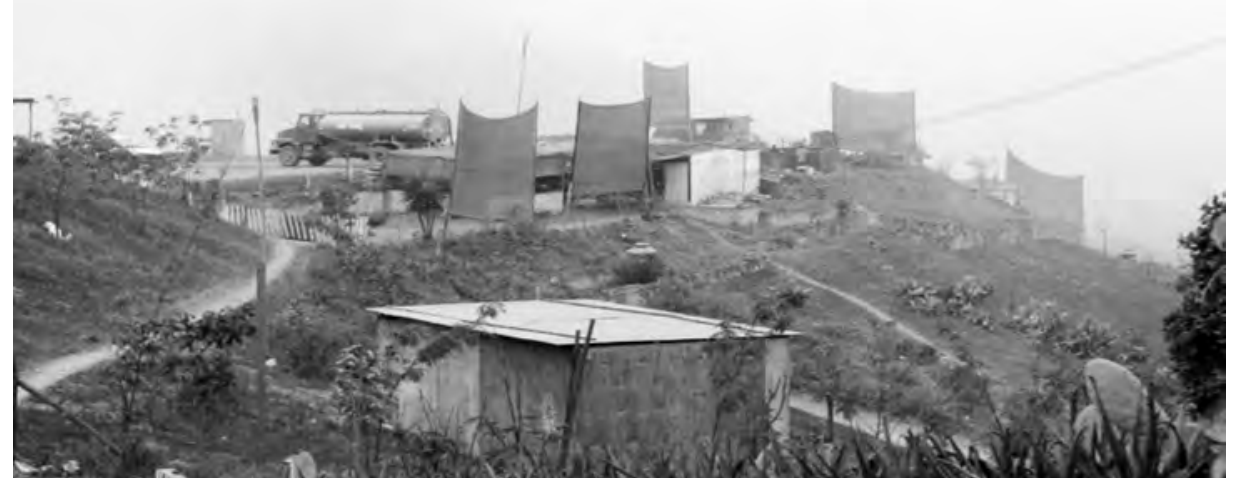

Figura 2: Atrapanieblas y su medio ambiente - Nueva Esperanza, Villa María del Triunfo. Fuente: Omar Mejía, Steven Alvarez y Edward Abarca, 2013. ${ }^{1}$

1 Agradezco a mis alumnos O. Mejía, S. Alvarez y E. Abarca por autorizarme a usar las fotos tomadas para el curso "Nuevas Audiencias y Escenarios de la Comunicación" que estaba a mi cargo en la carrera de Periodismo, en la Universidad de Ciencias Aplicadas - UPC. 
Ahora bien, de la Imagen 2 podríamos destacar instintivamente la condición de precariedad expresada en los caminos no asfaltados (solo trazados sobre la tierra), las viviendas de madera y techo de calamina y el camión trasladando agua a las alturas. En medio de la atmósfera húmeda, sin embargo, sobresalen cinco atrapanieblas que indican zonas intermedias, y que funcionan como prolongaciones materiales de 'nuevas esperanzas'. Nótese, además, el contraste que los atrapanieblas marcan respecto del camión cisterna. Mientras los atrapanieblas para sobrevivir ahora se tienen que conectar con la red de vecinos de Nueva Esperanza y sus actividades diarias, los cisternas suelen ser recordatorios de que la red de agua y desagüe no está conectada a ellos, es decir, que el poder se encuentra en otra parte.

Efectivamente, en Nueva Esperanza (distrito de Villa María del Triunfo) la ONG "Peruanos sin agua" hace algunos años viene trabajando en la implementación, funcionamiento y aceptación de los atrapanieblas 3. Podemos decir que después de una década, los atrapanieblas han ampliado su programa de acción, abriendo paso a un nuevo escenario de experimentación. En aquella localidad han sido instalados veinte atrapanieblas de $24 \mathrm{~m}^{2}$ con capacidad para capturar entre 300 y 360 litros al día. Teniendo en cuenta los costes de la malla, las canaletas, los codos, los tensores, el reservorio y el transporte, la instalación de cada uno de estos artefactos asciende a S/.500. El agua obtenida, si bien aún no es apta para el consumo humano, sí serviría para la siembra de papa, camote, lúcuma y sábila; es decir, tendría alguna conexión con la economía local.

En nuestro análisis, el relativo éxito que ha tenido este proyecto en Nueva Esperanza se deriva de dos factores: (i) los atrapanieblas han sido definidos como 'objetos-fluidos' (community-based) y (ii) y se ha mantenido en el tiempo una cadena de problema-solución que es básica para la experimentación (potencial de aprendizaje).

El proyecto tiene una serie de aliados económicos como USAID-Perú, el Fondo de las Américas y la propia municipalidad de Villa María del Triunfo. Estas alianzas han sido producto de negociaciones pasadas y actuales. Asimismo, estudiantes de la Universidad Nacional Agraria de La Molina se han encargado de realizar análisis de calidad de agua. Y los vecinos han sido involucrados en el funcionamiento y mantenimiento de los artefactos; lo cual

3 La información del caso de “Peruanos sin agua” es recogida del estupendo ensayo de María Pía Flores, realizado el año 2012 para el curso “Nuevas Audiencias y Escenarios de la Comunicación” de la UPC. En su ensayo María Pía describe la atmósfera socio-técnica de los atrapanieblas y su conexión con la población, a través de una entrevista a Abel Cruz, presidente de "Peruanos sin agua." Agradezco la autorización para usar su ensayo en este trabajo.

\section{8/ REVISTA DESOCIOLOGÍA 25}


ha requerido establecer un vínculo con sus asambleas a fin de re-distribuir responsabilidades. La experiencia ha enseñado que involucrar a los vecinos no tiene que ver con crear puestos de trabajo, es decir, con convertirlos en empleados; ni tampoco hacer de los atrapanieblas donaciones o regalos. Estas formas de interesamiento (habituales en empresarios y políticos) son ineficientes al poner el peso en un solo lado de la balanza: o el intercambio sin apego o la filantropía sin esfuerzo.

A diferencia, un objeto-fluido pone el peso sobre la re-apropiación, es decir, un punto de equilibrio muy sutil entre técnica y compromiso, dos formas simétricas de agregación (Yrivarren y Salomón, s/f). En un trabajo fundamental de los estudios de la ciencia y tecnología, M. de Laet y A. Mol (2000) definen los objetos-fluidos de la siguiente manera:

Because in travelling to 'unpredictable' places, an object that isn't too rigorously bounded, that doesn't impose itself but tries to serve, that is adaptable, flexible and responsive -in short, a fluid object - may well prove to be stronger than one which is firm. (2000: 226)

La fortaleza de los objetos-fluidos tiene que ver con que sus límites no estén claramente delimitados, aunque resulte paradójico que un objeto en cuanto tal no sea 'sólido'. Y es que ese grado de indefinición en su materialidad y sus vínculos sociales les permite desplazarse hacia lugares duros, donde artefactos muy limpios y estandarizados no sobrevivirían mucho tiempo. En la fluidez estaría la clave de la re-apropiación. Sin embargo, el reto de los objetos-fluidos es mantenerlos en funcionamiento en la adversidad, lo cual implica aprender a solucionar problemas prácticos y generar habilidades localmente. En otras palabras, implica no limitar el potencial de aprendizaje de la organización.

A continuación veamos de qué manera, la organización amplió el conocimiento sobre los atrapanieblas respecto de la experimentación llevada a cabo a inicios de 1990. En cuanto a las mallas raschel y con la finalidad de capturar más agua, los 'sin agua' han ido probando un modelo diverso de atrapaniebla tipo embudo, desviándose un tanto del estándar de una malla bidimensional (como una net de voley). En relación con el problema de la limpieza del agua, han instalado biofiltros en los ductos por los que viaja el agua, filtrando el polvo y la suciedad. En la misma lógica problema-solución, la experiencia enseñó que el mejor período para la captación de agua es la estación invernal. Y si se captura agua más o menos eficientemente, se necesita almacenes. Al 
no contar con un reservorio de agua para el almacenaje, se negoció con el Fondo de las Américas para instalar reservorios con geo-membranas (cuyo precio es ciertamente elevado). Los reservorios de cemento convencionales suelen honguearse y pudrirse demasiado rápido. Asimismo, la experiencia ha enseñado que si no se guarda los atrapanieblas en un lugar especialmente acondicionado, protegiéndolos de la luz del sol y la humedad del verano, los artefactos terminan por blanquearse y pudrirse. Incluso, para la instalación propiamente dicha de los atrapanieblas se requiere el desarrollo de habilidades. En su ensayo sobre "Peruanos sin agua”, María Pía Flores señala:

Lo del atrapanieblas no es tan simple como 'poner por poner'. Se tiene que estar girando durante aproximadamente 15 días este mismo aparato en un metro cuadrado y para ello se requiere la presencia de una persona que chequee cuántos litros capturó y cuál de todas las posiciones arroja un mejor rendimiento. (2012: $\mathrm{s} / \mathrm{p}$ )

Este aprendizaje colectivo del tipo acomodo y re-acomodo y enfocado en los objetos es un elemento empírico esencial para comprender la naturaleza ingeniosa de los atrapanieblas. El potencial de aprendizaje es, ciertamente, una unidad de medida útil para evaluar la escala de cualquier innovación. Para B. Latour, una buena innovación se ubica en un punto intermedio entre saber y no saber; hallar ese punto depende en lo fundamental de la calidad de la experimentación ${ }^{4}$. Permítasenos una cita extensa:

El dilema del innovador es muy conocido: cuando puede, no sabe; cuando sabe, no puede. Al principio de su proyecto, si todavía no conoce en lo más mínimo las reacciones del público, de los financiadores, de los proveedores, de los colegas y de las máquinas que debe combinar en conjunto para que su proyecto tome cuerpo, puede, sin embargo, muy rápidamente, modificar de arriba a abajo la naturaleza de sus planes para adaptarse a sus desiderata. Al final de su proyecto, habrá aprendido finalmente todo lo que habría tenido que saber sobre la resistencia de los materiales, la fiabilidad de los componentes, la calidad de sus subcontratistas, la fidelidad de sus banqueros, la pasión de sus clientes, pero ya no podrá cambiar ni un poco sus planes: muy tarde, ya están ahí moldeados en bronce. La astucia de la innovación consiste entonces en hacer que dos curvas se entrecrucen: la primera representa la adquisición

$4 \quad$ Recuérdese que la confianza, tal como la hemos definido aquí, también es un punto intermedio entre saber mucho y la ignorancia total. En otro trabajo, hemos sostenido la simetría entre el desarrollo de la técnica (en laboratorios experimentales) y la construcción de compromisos (con actores heterogéneos) (Yrivarren y Salomón, s/f). 


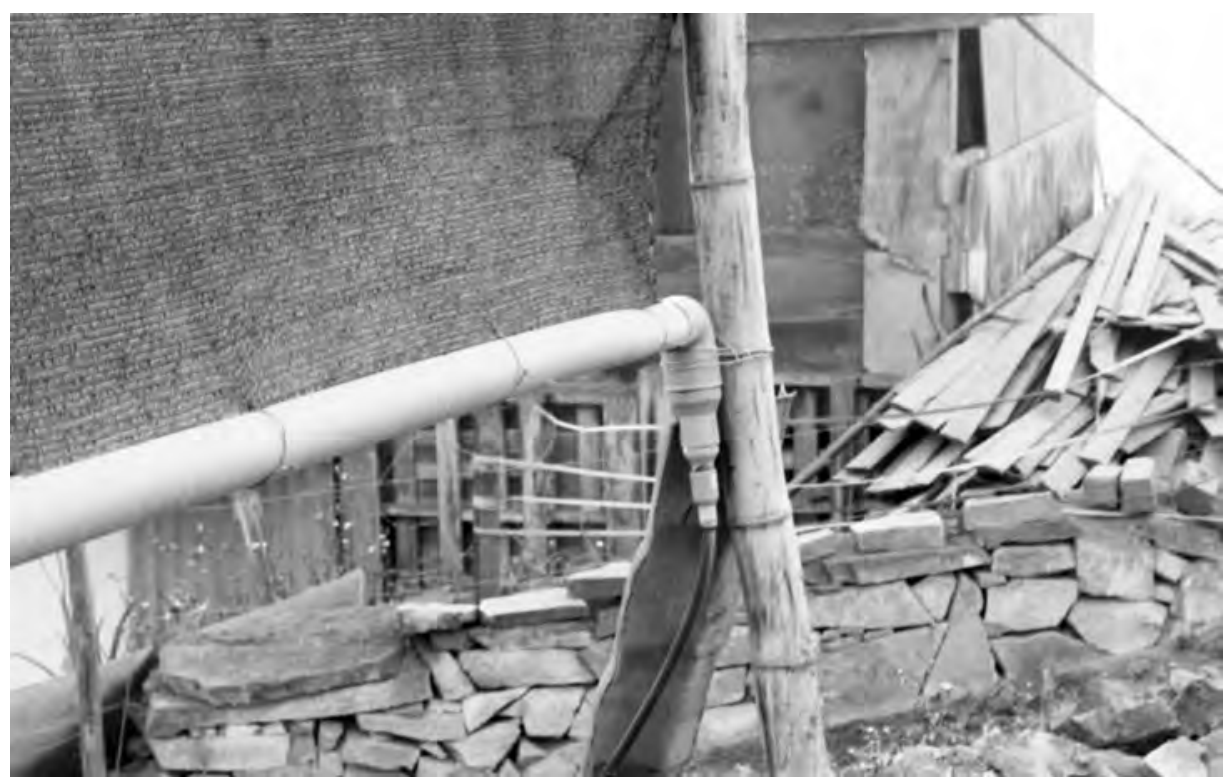

Figura 3: Detalle de un atrapanieblas, el codo de la canaleta - Nueva Esperanza, Villa María del Triunfo. Fuente: Omar Mejía, Steven Alvarez y Edward Abarca, 2013.

de los conocimientos sobre los estados del mundo; la segunda registra la pérdida de los grados de libertad del innovador. (Latour 2010: 233)

Consideramos que una evaluación del potencial de aprendizaje y la astucia de la innovación, en este sentido, es un indicador útil para cualquier estudio prospectivo. Dicha evaluación representaría el punto de encuentro entre el conocimiento de los mundos posibles (una mezcla de elementos sociales y no sociales) y las restricciones a la libertad de su creación. Para el caso de los atrapanieblas, la conformación de la red de actores involucrados, desde los inicios de la investigación experimental, dibuja un tránsito desde una tecnología intermedia hacia una tecnología community-based.

Sin embargo, no podemos decir que los atrapanieblas ya hayan sido “moldeados en bronce"; su experimentación de mundos posibles continúa. Volvamos al premio Juan Günther de marzo de 2014, con el que iniciamos esta sección. El proyecto "Parque Atrapanieblas”5 plantea un nuevo dibujo socioambiental de la quebrada El Paraíso en Villa María del Triunfo en base a la

$5 \quad$ No tenemos evidencias de que exista alguna conexión directa entre el proyecto de "Peruanos sin agua" y el de "Parque de Atrapanieblas". Lo único que sabemos es que los "sin agua" no han tenido un vínculo fluido con la Municipalidad de Lima. 
incorporación transversal de atrapanieblas. Se trata de un parque compuesto por: áreas de almacenamiento y distribución de agua, biodigestores para la producción de gas, áreas de recreación y descanso, planta de reciclaje, humedal artifical y plantaciones de sábila. Bajo un diseño diferente de las mallas clavadas en el suelo de Nueva Esperanza, aquí los atrapanieblas adoptarían la forma de 'barandas' ubicadas a lo largo de todo el recorrido del parque. Las mallas-barandas iniciarían el ciclo del agua, que pasaría luego a una acequia que, a su vez, alimentaría un sistema de riego por goteo. Hagamos notar que los atrapanieblas ahora forman parte de una composición diferente del espacio urbano, que enlaza naturaleza y sociedad de un modo alternativo. Este cambio en la 'composición' es el que hace más simple representarnos el tránsito del ingenio a la innovación.

El diseño del parque también imagina y describe un mundo posible. Su finalidad es revalorizar el entorno natural de las lomas, así como impedir el crecimiento urbano por invasiones promovidas por traficantes de terreno. Pero, a diferencia del estilo habitual, impedir el crecimiento urbano no conlleva limitar la libertad individual de los vecinos poniendo muros o desplegando policías, sino ampliar la franja verde con ayuda de los atrapanieblas.

En el lenguaje arquitectónico, esto significaría incorporar 'bordes' en el progama de acción de los atrapanieblas. Un borde es sobre todo un instrumento más que un simple concepto. Cuando entre dos zonas o espacios hay un borde, estos no están separados, sino que comparten límites suficientemente porosos para facilitar buenas combinaciones (en este caso entre lo social y lo natural) (Augé 2010, Sennett 2012).

En fases sucesivas se establecerán recorridos donde se hará destacar en primer lugar el borde entre el parque y el área urbanizada. Una vez culminado este, el camino envolverá los asentamientos de modo tal que indicará el borde entre la ciudad y el paisaje natural.

[...]

Poco a poco las zonas verdes se unirán hasta formar una cinta verde que acompañará todo el recorrido conviertiéndose en un borde de sí mismo. (Ege 2014: 22)

De este modo, la incorporación del proyecto arquitectónico hace más compleja la composición de los atrapanieblas en el distrito. No es el caso que se haya pasado de 'algo hecho por la gente' a 'algo hecho por expertos', o sea, no es que se haya dado un salto entre el conocimiento práctico al conocimiento experto. Esta forma de interpretar la situación es inútil, pues solo acrecienta 


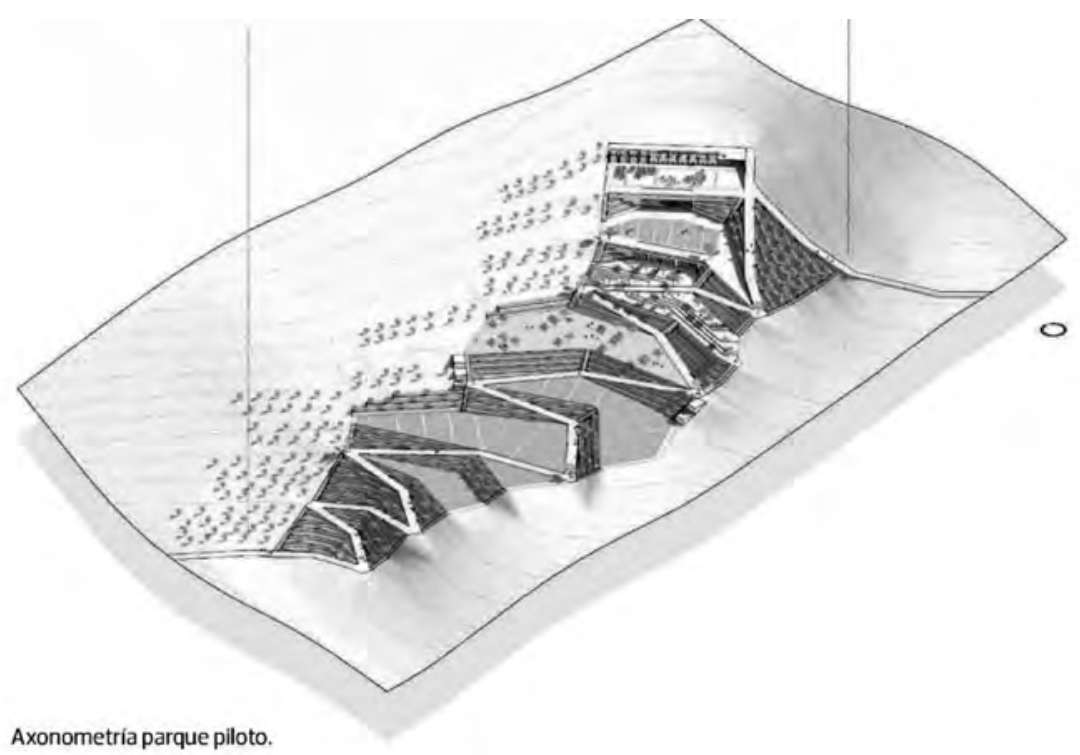

Figura 4. Parque atrapanieblas. Fuente: Revista Arkinka, nº 220, 2014, p. 20.

la desconfianza y el divorcio entre acontecimientos y escenarios. Más bien, lo que sucede es una ampliación de la zona intermedia; pero ahora entendemos mejor como funciona esa amplicación: mientras más acontecimientos incorporamos, mejores escenarios somos capaces de elaborar.

\section{Conclusión}

La idea más simple que organiza este ensayo es que, así como imaginar escenarios futuros para tomar decisiones hoy es importante, también lo es decidir cómo y desde dónde fabricaremos tales escenarios y mundos posibles. Esta idea resume la necesidad de sintonizar acontecimientos y escenarios, o como también nos hemos referido, sintonizar ingenio e innovación en el Perú. En nuestra lectura, el equilibrio entre lo que parece dos extremos irreconciliables puede lograrse si los escenarios son reconocidos en los acontecimientos y sus relaciones, y la innovación es asumida como efecto de ampliación de los oficios y artefactos ingeniosos. Este equilibrio es importante para asentar un clima de coherencia y respaldo al rededor de los proyectos individuales y colectivos que dan vida al trabajo cotidiano. Es más, consideramos que es un requisito indispensable para fortalecer un tiempo institucional.

En términos prácticos, se puede iniciar una fructífera discusión prospectiva poniendo entre paréntesis la certeza y la ansiedad. Así, creemos útil salir de la 
retórica del “así van a ser las cosas, ¿estamos preparados?”, y comenzar una conversación pública en torno a ‘zonas intermedias' o, lo que es lo mismo, en torno a datos portadores de (poco) futuro. La particularidad de estos datos es que ayudan a vislumbrar, a lo lejos e imperfectamente, mundos deseados y posibles; y ello gracias a que dejan traslucir su redes de producción de conocimiento.

En nuestro análisis sobre los atrapanieblas en Villa María del Triunfo, hemos intentado describir el tránsito del ingenio a la innovación a partir del seguimiento de datos portadores de poco futuro. El mundo deseado de los atrapanieblas al inicio fue la reforestación de las lomas costeñas y el aprovechamiento del agua para las cosechas y, en el mejor de los casos, para el consumo humano. La característica más destacable del proceso que va desde inicios de la década de 1990 a la actualidad es que los atrapanieblas han existido como 'objetos-fluidos', es decir, como artefactos imperfectos por definición; y, en consecuencia, su funcionamiento y mantenimiento están sujetos a una cadena problema-solución, que asociamos al potencial de aprendizaje en la organización. En otras palabras, el mundo de los atrapanieblas vive de la experimentación, adaptación y re-apropiación. Fuera de este ecosistema, los atrapanieblas no dicen nada del futuro. Como muchas cosas en el Perú, estos artefactos no nacieron de un plan o escenario racional ni de unos medios eficientes para su realización. Por el contrario, en la experimentación misma los escenarios fueron emergiendo.

Por ello, consideramos que una evaluación más atenta de los objetosfluidos y el potencial de aprendizaje puede incrementar el conocimiento de los estudios prospectivos. Es más, puede conducir a una mejor interpretación de la prospectiva como una habilidad social. En el caso de los atrapanieblas, notamos que el desarrollo de la imaginación es el correlato de la conformación de una red de actores más amplia. Inicialmente, dichos artefactos fueron parte de experimentos a pequeña escala realizados por profesionales e ingenieros, luego pasaron a manos de 'los sin agua' y se transforman en artefactos community-based $\mathrm{y}$, finalmente, observamos que fueron incorporados dentro de un proyecto urbano más amplio al intervenir arquitectos y diseñadores.

La imaginación de mundos posibles se enriquece, por lo general, gracias a su propio potencial. No hay necesidad de apurar escenarios, menos aun en desconexión con los acontecimientos. Las trayectorias de innovación más ricas, complejas e interesantes son aquellas que nos hacen más sensibles a nuevas mezclas ingeniosas y a nuevas articulaciones de acontecimientos.

\section{4/ REVISTA DE SOCIOLOGÍA 25}




\section{Referencias bibliográficas}

Augé, M. (2010). La comunidad ilusoria. Barcelona: Gedisa.

BAS, E. (2004). Megatendencias para el siglo XXI. Un estudio Delfos. México: FCE.

BITAR, S. (2014). Las tendencias mundiales y el futuro de América Latina. CEPAL InterAmerican Dialogue.

Ege, M. (2014). Parque atrapanieblas. Arkinka - Revista de Arquitectura, Diseño y Construcción, año $18, n^{\circ} 220$.

Flores, M. P. (2012). El caso de los Peruanos Sin Agua y una historia de atrapanieblas en Villa María del Triunfo. (Ensayo presentado al curso Nuevas Audiencias y Escenarios de la Comunicación). Lima: UPC.

Latour, B. (2010). Crónicas de un amante de las ciencias. Buenos Aires: Dedalus.

LeAt, M. y MoL, A. (2000). The Zimbabwe Bush Pump: Mechanics of a Fluid Technology. Social Studies of Science 30(2), pp. 225-63.

MÁrqUEZ, J. (1993). Atrapanieblas. Una tecnología para la reforestación de las lomas costeras del Perú. Ingeniería Industrial, 2(7).

RORTY, R. (2002). Filosofía y futuro. Barcelona: Gedisa.

Pinche, C. y Ruiz, L. (1995). Captación y aplicación del agua de niebla en las lomas de Atocongo. Alma Máter - Revista de Investigación de la Universidad Nacional Mayor de San Marcos, $\mathrm{n}^{\circ} 10$.

Nugent, G. (2005). Vindicación del ingenio. Acerca de la vida dura y el crecimiento moral. (Manuscrito inédito).

SenNett, R. (2012). Juntos. Rituales, placeres y política de cooperación. Barcelona: Anagrama.

Simmel, G. (1972). El secreto y las sociedades secretas. En Simmel, G. Sociología 1. Estudios sobre las formas de socialización. Madrid: Alianza Editorial.

YRIVARREN, J. y SALOMón, J. (s/f). ¿Por qué no funcionó? El dilema entre técnica y compromiso. El caso de la siembra directa en el Perú. (Pre-print) Ciencia, Público y Sociedad, $\mathrm{n}^{\circ} 2$. 